\title{
LA RECONSTRUCTION DES DROITS HUMAINS D'APRÈS CELSO LAFER:POINTS DE REPÈRE POUR L'ÉTUDE DE LA PROBLÉMATIQUE DE LA RUPTURE TOTALITAIRE ET DES DROITS HUMAINS
}

\author{
Eduardo Carlos Bianca Bittar \\ Aluno do Curso de Graduação da Faculdade de Direito da USP
}

Resumo:

O pensamento de Hannah Arendt em torno da ruptura totalitária, assim como em torno das atividades fundamentais da vita activa e da vita contemplativa, sob as luzes das premissas helenistas, e dentro de uma perspectiva existencialista, constitui fecundo material para a reflexão contemporânea acerca do problema que indica a natureza do homem como ser social: os direitos fundamentais.

Résumé:

La pensée de Hannah Arendt autour de la rupture totalitaire aussi qu'autour des activités fondamentales de la vita activa et de la vita contemplativa, sous les lumières des prémisses hélénistes, et dans une perspective existencialiste, constitue féconde matière pour le raisonnement contemporain sur le problème qui prend l'homme comme un être social: les droits fondamentaux.

Table de matières:

1. La survenance de la philosophie du droit.

2. Le totalitarisme et la rupture historique.

3. Hannah Arendt: le discours et l'importance de l'espace publique.

4. Displaced persons et le droit à avoir des droits.

5. La naissance des droits fondamentaux et leur évolution.

6. La pensée, les principes et les réfléxions arendtiennes.

Bibliographie.

1. La survenance de la philosophie du droit.

L'organisation des hommes en société est un phénomène politique qui se mélange avec les normes de la morale, des coutumes sociaux et des préscriptions juridiques. Dès le début des temps de la pensée, le problème fondamental de toutes les recherches rationnels qui a fait l'être humain se tourner vers les questions 
ontologiques, ces genres de questions qui remettent le sujet comme le seigneur des découvertes plus intrinsèques des choses. C'est ne pas faux de dire que le but de toute investigation ontologique est l'essence de l'objet recherché.

Dans le domaine de la pensée juridique, des paradigmes ont été formé dans la course temporel de l'histoire, conséquence directe des élaborations philosophiques des penseurs qui cherchaient la raison d'être du Droit, autant que science et autant que norme de conduite social. Un des ces paradigmes multiseculaires est celui du droit naturel, venu de la tradition conceptuelle aristotélique du juste naturel (díkaion physikón) et qui se fait toujours présent à travers la dicotomie nomos-physis. Historiquement, l'idée de droit naturel s'est originé de la nécessité de laïcisation dans le contexte de rupture avec la pensée médiévale, représentant l'idéal d'imutabilité caractéristique de la science du droit. À son nom est toujours liée la conception d'une loi supérieur et qui est au delà de la mutation et de la pluralité de l'éphemère.

Dans cet ordre d'idées on peut détacher quelques lignes fondamentales de l'histoire du paradigme de la philosophie du droit autant que résultat de l'expérience historique. Ainsi, d'abord, ce qui a été conçu comme juste naturel dans le contexte de l'Antiquité, et qui a été reçu par la tradition médiévale, ensuite, devient le model moderne de Droit naturel depuis l'avènement des travaux rationalistes de $\mathrm{H}$. Grotius. La raison réfléxive, qui apparaît comme valeur central de tous les raisonnements modernes, a introduit la notion de doute dans le domaine de la connaissance humaine, ce qui a été suffit pour ouvrir les chemins de la recherche des fondements du Droit hors des domaines de la foi réligieuse et de la révélation divine de la vérité. Le travail cientifique, à ce temps-là, a commencé à détruire les dogmes de la connaissance et à les changer par des postulats qui composent l'ensemble de ce qu'on appelle gnoséologie. Les prémiers pas vers la dissolution de l'idée du droit comme phénomène fondé sur une raison universelle naturelle où sur des arguments notamment réligieux n'avait plus d'espace dans le corps des travaux de la pensée depuis le commencement d'une nouvelle période historique,celle de la modernité.

La positivation des droits humains, pendant le XVIIIème siècle, a contribué pour renforcer la thèse de la relativité historique de ce qui était conçu comme droit humain par nature, une fois que le siècle XIX apporte des nouvelles révendications essentielles, en faisant que la raison n'accèpte plus le concept, ce qui le fait proprement replonger dans l'obscurité de ce que l'on conçoit comme mythique 
dans le contexte des sciences juridiques, pour fonder les études sur une nouvelle conception épistémologique; c'est formé le paradigme de la philosophie du droit, notion qui est venu pour déplacer le concept déjà surpassé du droit naturel.

Actuellement, la philosophie du droit est vue comme le produit de la pensée des juristes preoccupés avec le développement d'une science batît sur l'imaterialité d'un idéal métaphysique indeterminé. Ainsi, la philosophie du droit n'est pas un étude unitaire et singuralisé par la vision limitée de la réliaté. Par contre, il s'agit d'une science qui se répandit en se pluralisant par la complexité de ces cotés d'études. Alors, des subdivisions didactiques, nécessaires en fonction de l'espécification et de l'aprofondissemnt des abordages scientifiques, peuvent relever la méthode, la forme des lois, le fait et la société, où l'idéal de réalisation du valeur $\mathrm{du}$ juste, ce qui constitue proprement des rames de la philosophie du droit, respéctivement, méthodologique, ontologique, sociologique et deontologique.

Le questionement sur la position et le rôle de la philosophie du droit dans le contexte actuel de développement de la téchnique scientifique juridique se détache comme la fonction essentiel du juriste integré aux plus évidents et plus importants changements sociaux. L'univers de la recherche autour du significat du Droit, de son essence, de son telos, s'ouvre à nouveau devant les régards de ceux qui pensent et vivent les problèmes de la pratique juridique dans la complexité historique dans laquelle les valeurs de la culture se rencontrent.

La proposte d'étude de la reconstruction des droits humains à partir des expériences vécus au cours du XXème siècle, siècle où toute l'humanité a dû subir deux conflits mondiaux, intègre non seulement la nécessité d'analyse des événements actuels et de ces conséquences pour l'homme, mais aussi l'idée de critique scientifique de la réalité aux rôles fondamentaux de la philosophie du droit, pensée de la pensée juridique. Si le Droit est un produit culturel social, et si la science du droit s'occupe de son développement, sa philosophie ne peut qu'atteindre son telos propre en poursuivant les questions qui ont lieu dans la mutation historique de la réalité. L'étude autour de la question de reconstruction des droits humains ne s'éloigne pas de ces objectifs.

2. Le totalitarisme et la rupture historique.

Des notions d'harmonie linéaire, de procès historique, d'évolution systématique traversent tous les raisonnements qui ont travaillé le problème du Droit 
jusqu'au XXème siècle. Tout était conçu comme conséquence d'un phénomène naturel de causalité qui permet la prévision des conséquences et des effets. Celà n'est qu'une forme d'établir une norme de controle sur la pluralité des faits historiuqes, le plan, par excellence, de l'infinit et de l'indétermination. Des nouveaux questionements ont été fait nécessaires dès le moment qu'un coup historique s'est produit au cours du XXème siècle: le totalitarisme.

La rupture historique qui s'est produit en conséquence de l'avènement de l'ideologie totalitaire est la préocupation central des études arendtiens. D'ailleurs, basé sur l'ordre juridique caractérisé par l'amorfisme structurel, ainsi comme sur la nebulosité de la sisthématique burocratique organisationnel, le régime du totalitarisme a été précisement l'élément de désordre dans l'idée de rationalité liberal qui était le corolat de toute la pensée depuis la Revolution Française et l'ascension des principes bourgeois. Le totalitarisme s'est répandu en se baseant sur le modèle positiviste du droit, qui faisait de la loi l'instrument de pouvoir capable de consentir la reductio ad Hitlerum. Le pouvoir se justifie à travers la loi, étant donné que ce qui est établit originairement par la volonté de l'État devient légitime dans le contexte de la légalité; désormais, la loi perds ses fonctions originelles de moyen d'organisation de la société et de garantie d'un système basé sur les principes démocratiques de conservation de droits et de participation légitime dans les décisions politiquejuridiques.

Dans cet ordre d'idées, le totalitarisme s'est instalé en détruisant la logique du raisonable historique; plus de causalité, plus d'effets: dès l'avènement du systhème totalitariste, tout est possible. En faite, la régression totalitaire a surpassé des siècles de traditions juridiques et s'est imposé par la force de l'arbitraire, ce qui signifie non seulement un coup historique, mais aussi l'ascension et l'aparition d'un model absolument originel dans le contexte des études des sciences politiques, étant donné que le régime totalitaire n'a rien à voir avec l'absolutisme et la tiranie. Les progrès téchnologiques du XXème siècle, des traits qui prouvent l'ascension de la téchnique et de la raison apliquée à la production et à la vie pratique, se contradisent avec l'intervention d'un système de gouvernement basé sur l'irrationalité de la force arbitraire.

Dans le contexte d'émergence de l'idéologie du pouvoir totalitaire, la personalité humaine disparaît, parce que le système, à travers l'établissement d'une peur générale et constante dans la société, réduit les idées de participation publique et de vie privée aux figures de la méfiance et de la suspéction. La préssion, ce jeu de 
nerfs, massacre la libérté plus éssentiel de l'être: celle d'être soi-même dans le contract social. La torture ménace tous les consciences et réduit la personnalité à ce que le régime veut qu'elle soit.

3. Hannah Arendt: le discours et l'importance de l'espace publique.

Tout dialogue avec les idées de Hannah Arendt détache le discours comme le plus important méchanisme de participation et d'actuation sur la réalité, pensée notamment traversée par le model grec d'organisation démocratique. Les valeurs de la communication, de l'agremiation et de la participation dans la pluralité constituent les points centrals du contact publique humain. Toute société libéral se fonde sur la prémisse constitutive qui résulte de l'utilisation du logos, raison fondante de toute l'esphère publique et de toute la complexité des rélations humaines, comme source de toute réalisation humaine qui puisse vinculer à tous. L'importance de la manutention de l'ouverture participative de l'espace publique implique des conséquences sur le concept de légitimité; le pouvoir ne seras légitime que quand il s'affirme comme conséquence d'une décision prise en accord logistique par la pluralité des agents en publique.

La sphère publique, dans la pensée arendtienne, est le lieu de l'areté inhérente à ceux qui sont naturelement disposés pour la vie politique: les êtres rationels (physei politikón anthropos). Le principe aristotélique qui détache l'argument de la formation teleologique de l'homme pour la vie dans l'espace commun se rélatione intrinsèquement avec l'évidence fénomenologique de l'importance de la parole, écrite ou parlée, pour la communication entre ceux qui partagent l'ambience social. Le publique, diféremment du privé, est caractérisé, ou bien doit être caractérisé, par les éléments de la visibilité, de l'apparence de tout ce qui potenciellement peut être transmit aux autres, et de la structure mondaine artificielle qui est le produit des travaux humains et qui conditionnent l'existence sur le monde. La préocupation arendtienne se base sur le problème de la pluralité des hommes, capables de interchanger des informations à travers le contact humain du langage rationel; dans la normalité du fonctionnement de ce phénomène, l'homme se revèle comme individualité qui peut se montrer aux autres par moyen des manifestations de communication, dans le sens classique du terme (action en commun). 
Il faut souligner que la limitation existenciel de l'homme sur le monde fait que la mortalité soit le point arquimédien de toute l'éspeculation métaphysique, ainsi comme la caractéristique central de la condition humaine. Néanmoins, la circunstancialité de la vie humaine sur le monde ne fait pas de l'homme un être anonime, parce qu'il est toujours possible que l'individu laisse un morceau de son expérience dans la mémoire des générations. Cette capacité de se faire présent aux autres à travers ces actes donne une certaine durabilité à la notion classique de vie (de la naissance jusquà la mort physique) et permet que quelques faits deviennes imortels et qu'ils rencontrentconstitue dans l'espace publique (agorá dans l'Antiquité) son lieu caractéristique enrte les hommes.

Une autre évidence se détache pour renforcer l'autorité de l'argument qui est pour une forme de vie qui permet le contact publique: rien sur le monde est fait pour une seule génération; il reste toujours implicite dans toutes les activités humaines, surtout dans la plus futile de toutes, la labeur, l'idée de durabilité du monde et des créations humaines qui peuvent être transmises à travers le temps, d'où on peut parler d'un héritage culturel, d'un héritage politique, d'un héritage juridique, téchnologique... Tout ce qui appartient aux domaines du mondain conditionne l'homme et, étant donné que l'homme, autant que genre, n'a pas un limite d'existence, les faits sociaux, les actions, les progrès téchnologiques, l'évolution de la science et de la pensée, la morale, les habitudes, les traditions s'éternisent dans une succéssion jusqu'à l'infinit des générations humaines.

Mais le totalitarisme est venu pour prouver qu'il était capable de détruire la vie publique, ainsi comme d'éliminer la vie privée, par la régression de la participation qui soutient la société. L'opression instale le régime de la peur, en cachant la vérité et en détruisant la communicabilité inhérentes à la vie publique, éléments fondamentaux de la subsistence de la communité d'après les conclusions arendtiennes. L'extrémisme révolutionnaire a rendu surprise toute la société civilisé du XXème sièclé, société celle qui se baseait sur les postulats de la cohérence juridique et de la prévision naturaliste des faits. Rien dans la modernité n'a mieux prouvé l'idée de que les droits humains ne sont que des fruits de l'actuation de l'homme dans le monde social que le totalitarisme, une fois que tout est possible dans l'idéologie du régime. 
4. Displaced persons et le droit à avoir des droits.

Une autre importante conséquence des totalitarismes nazi et stalinien a été la création de ceux qu'on appelle displaced persons, les anonimes qui, une fois marginalisés par l'incohérence idéologique d'un système d'opression, n'ont rencontré aucun lieu qui puisse les avoir comme des citoyens. La condition de ces personnes s'est originé d'un mélange de problèmes économiques, sociaux et politiqueidéologiques entre les deux guerres mondiales. Pourtant, dans l'histoire de la pensée, les droits ont été toujours attachés aux principes intrinsèques de l'éxistence d'un pouvoir organisé qui puisse les sauvegarder aux individus. La fissure de cette erreur est apparue exactement au moment que des grandes masses de personnes ont été réduites à des organismes inutiles pour le monde, une fois qu'aucun État ne pouvait déffendre, juridiquement, ces droits contre l'opression et la torture du système totalitaire. Le point faible de toutes les théories libéralistes était découvert... La conclusion fondamentale de toute cette expérience détruit le model paradigmatique construit par la tradition jusnaturaliste grocienne; les droits humains sont intrinsèquement attachés à l'idée de status civitatis, une fois que tous les droits dérivent de la convention humaine et dépendent directement d'un système de lois d'un État qui se détache pour la protéction de l'individu.

Ces groupes d'anonimes qui ont été exclu de l'ordre national, ce qui a originé la formation de masses humaines sujettes au génocide, crime qui n'était pas prévu par le droit international et qui a rendu stupéfaits les personnes qui pouvaient prévoir les conséquences de ce régime. Ceux déplacés se sont vus comme des êtres inutiles sur le monde, une fois qu'aucune loi, qu'aucun système, qu'un aucun pays pouvait les considérer comme des sujets de droits. La citoyenité s'est montré une conditio sine qua non pour l'éxercise des droits humains; la prémisse imanentiste de l'éxistence des droits inhérents aux hommes s'est montrée aussi fausse que l'idée de la prévisibilité historique des phénomènes. La réaction n'a pas tardé. Le genocide a commencé a être conçu comme un crime contre l'humanité; il était très vif dans la mémoire humaine pour qu'il puisse avoir été facilement oublié.

5. La naissance des droits fondamentaux et leur évolution.

La philosophie politique, et encore humaniste, arendtienne s'est directement formée sur la conscience d'une nouvelle découverte, celle de que tout est 
possible et que le futur peut apporter des nouvautés inattendus. Le problème de l'imprévisibilité du futur a désormais donné le coup d'éclat nécessaire pour que des providences puissent être prises en raison de l'idée de prévention. Le droit à avoir des droits est un des fondéments de toute la réfléxion arendtienne, ce qui est devenu la question fondamentale des discutions de l'après-guerre.

Les conquêtes juridiques ont été faites à partir de l'expérience totalitaire de la IIème guerre mondiale. Les premières réponses aux problèmes du génocide et des displaced persons datent de 1954 et de 1961 et encore d'autres les ont suivis. La nécessité d'exorciser le fantôme de l'indétermination de l'apparition d'un autre coup d'État fondé sur des bases totalitaires n'a que suscité le problême des droits humains dans l'esphère du droit international, d'où des divers questionements sont venus.

Quant à ce qui concerne à l'origine des droits humains, progressivement, dans le contexte de l'évolution de la pensée humaine sur l'homme autant qu'homme, on peut distinguer des très sensibles pas vers l'affirmation de ce qu'on admet par droits fondamentaux. Premièrement, les Écritures et l'égalité, la philosophie stoïcienne et l'univers humain, les postulats chrétiens, l'individualisme et la réfléxion moderne sur l'ego, ainsi comme les conflits réligieux du XVIIème siècle, ont contribué pour former l'esprit humain autour de la question des droits fondamentaux. Deuxièmement, la sécularisation et la rationalisation de la culture ont donné le contribut final pour le procès de positivation de ces droits au cours du XVIIIème siècle avec les Révolutions Américaine (1776) et Française (1789). L'homme, dans cette période, avait déjà s'affirmé comme le maître de son propre destin, le seul responsable pour la conduction de son histoire et de son développement. Non plus l'objet passif de l'histoire, mais le sujet actif de tout le procès de changement du social. D'abord le cartésianisme et le problème subjectif de la connaissance du cogito, ergo sum; ensuite, le contratualisme roussaunien, qui n'a été que le signe plus caractéristique de cette pensée antropocentriste.

On peut distinguer la formation d'une première génération de droits qui s'est imposé dans une perspective d'opposition aux pouvoirs étatales au cours du XVIIIème siècle. L'individu est le sujet des droits fondamentaux contre l'opréssion royale. Le contexte était celui de l'illuminisme et les idées n'étaient pas plus fécondes que celles de défendre l'esphère de l'individu de l'abus de pouvoir. L'actuation du gouvernement ne serait différente que celle d'abstention; c'est la liberté négative, ne pas être bousculé par les mains du pouvoir, parce que l'économie marche par elle 
même comme conséquence de l'impulse des forces invisibles du marché, selon le model doctrinaire de Adam Smith.

La survenance de divers changements sociaux emmènent le problème de la justice distributive s'est rapidement posé, et la governabilité a été dificulté par le répandissement des questions sociales. C'était plus le cas d'avoir des droits comme garanties positivées, mais le cas d'avoir les conditions de répondre aux nécessités élémentaires de la vie, comme la santé, le loisir... Les charges et les avantages sociaux n'étaient pas très bien partagés dans la société, ce qui a fait émergir des problèmes encore de raison individuelle, mais, maientenant, caractérisés par le domaine de la nécessité. La Révolution Industrielle avait produit ces premières éffets dans l'escale social; ces marques s'ont fait sentir sur les ouvriers, le seul éloigné des richesses produites pendant le processus d'acumulation primitive et de prodution en escale industrielle par l'avènement des téchniques bourgeoises de production. Les premiéres garanties surgent au cours du XIXème siècle (Constituition Française de 1848), mais l'affirmation mondiale des conquêtes acquéris par l'éxercice de la grève et de la manifestation ouvrière n'a été fait qu'au cours du XXème siècle, avec la construction des postulats constitutionalistes sur ces droits, aussi fondamentaux que les droits de première génération, malgré le manque de prévision des penseurs liberaux.

Une troisième, et aussi une quatrième, ligne de droits apparaît dans le contexte du monde contemporain, où se détache l'idée de colectivité, de groupe, de famille, d'humanité. L'ONU est l'organ responsable par la légitimation et l'affirmation de cette nouvelle construction social, dans le sens que les droits sont les produits de l'évolution historique de l'homme dans la vie sociale, qui cherche la protéction des valeurs de la paix, du développement des nations, de l'environement, des fonds océaniques comme patrimoine commun de l'humanité... Les questions ne se réduisent plus à des sujets individuelles, se détachant, tant de fois, de l'ambience régionel d'un État pour atteindre le status de problème humain, mondiale. Les frontières ne limitent plus les droits et les questions d'aspect global émergent ayant comme conséquence l'affirmation de la falibilité de l'aplication de la conception individualiste des droits dans le monde contemporain.

Ce procès évolutif des droits dans le contexte juridique, constitutionel et international, ne font que périr le model libéral des droits humains. Toute l'arbitraireté de l'affirmation jusnaturaliste se réduit aux contingences du changement pour qu'on puisse admettre le principe de l'historicité de la construction de justice, 
soit au plan des droits fondamentaux, soit au plan du droit conçut comme système de réalisation de l'ordre social.

6. La pensée, les principes et les réfléxions arendtiennes.

Les clés de la pensée de Hannah Arendt sont des prémisses hélénistes qui accentuent la nécessité de perféccionement de la vie démocratique, parmi lesquelles on détache: la transparence de l'actuation politique, idée qui détruit le dogme déterministe qui atribue au pouvoir la capacité de corrompre; visibilité de tous les procès qui sont dans le domaine du publique; intersubjectivité, essence de l'actuation humaine et de la reálisation de la nature humaine, selon les orientations aristotéliques; participation, valeur fondant de la construction de la société véhiculé par le pouvoir de l'archein (actione), ce qui représent l'initium de tout procès sociohistorique.

D'une part c'est l'aversion à toute obscurité, à tout ce qui est secret et qui se cache des yeux du jugement publique. Aux idées d'opression et de peur, établis par la difusion du mensonge et de l'incertitude burocratiques, le système arendtien oppose les idées classiques qui fondent l'État batît sur le model d'organisation juridique et démocratique. D'autre part il ne s'agit que d'un retour, dans le sens de reconstituition ou de reconstruction, mais, de toute manière, un refaire le présent et un construire le futur sur les principes héléniques de liberté, publicité et participation. Enfin, c'est une pensée qui retablit l'état et la fonction naturel de l'art politique dans le contexte de la modernité.

La synthèse des idées arendtiennes ne témoigne que la nécessité de remise en question de toute la vie moderne. Une diversité énorme d'arguments peuvent êtres éloignés comme preuve de celà, mais il ne faut pas beaucoup pour montrer que tout un héritage intellectuel s'est déjà formé autour des fécondes réfléxions et des prémisses qui ont bousculer les thèmes de la politique, de l'existencialisme, de la subjectivé de l'esprit humain, parmis d'autres.

Au même temps qu'on faisant une apologie de l'action (vita activa) et une aplication philosophique pour l'humanité, Hannah Arendt ne nous a pas laissé un corps théorique inanimé pour que l'on fasse vivre à nouveau. Le mouvement lui est inhérent, une fois que ces concéptions sont en plein accord avec les problèmes de la modernité. Métamorphoser sa pensée est surtout établir un dialogue avec ses idées, 
avec son oeuvre; celà permet proprement qu'on y rencontre potenciellement la substance pour composer des arguments pour l'être humain dans sa pleine nature.

Toute bataille pour les droits humains ne s'écarte pas d'affronter la proposte qui pose d'autres questions fondamentales, telles comme, selon Celso Lafer dans ces conclusions sur la thématique:

a.) la citoyeneté doit être conçue comme le droit à avoir des droits, en vue de la question suscitée par les displaced persons dès les conflits mondials;

b.) la nécessité de repression du génocide comme crime contre l'humanité entière;

c.) les droits d'association et de résistence civile contre l'opression de l'abus de pouvoir et de la supression de droits;

d.) le droit à l'information, rapport fondamental pour la subsistance d'une sphère publique éclairé par la force du libre discours.

Sur le même plein, la bataille pour la conquête des droits humains ne se relève que comme un tour vers l'intérieur de l'homme, dans sa subjectivité, ainsi comme vers son extérieur, dans sa capacité d'établir des rélations socials, recherche qui se montre à nos yeux comme incessante. D'un univers restrict de prémisses dans la Déclaration des Droits de l'homme et du Citoyen de 1789, enconcées par l'article II ("Le but de toute association politique est la conservation des droits naturels et imprescriptibles de l'homme; ces droits sont la propriété, la sûreté, et la résistence à l'opression"), jusqu'à une multiplicité de garanties essentielle dans la contemporanité, la problématique ontologique humaine ne se montre que toujours présente. Cette présence suscite des réfléxions qu'à partir d'un model philosophique peuvent être faites sous la perspective de l'homme autant qu'essence. En résumé, les thèmes de la liberté, de la participation, des droits fondamentaux n'ont qu'une seule cellule en commun pour les donner l'unité, à savoir, l'homme, celui-ci qui a été, véritablement, le point central de toute la recherche arendtienne. 
Bibliographie

ARENDT, Hannah. Origens do totalitarismo. Trad. de Roberto Raposo. $2^{\mathrm{a}}$ ed. Rio de Janeiro : Ed. Documentário, 1979.

ARENDT, Hannah. The human condition. Chicago : The University Chicago of Press, 1958.

ARENDT, Hannah. The life of the mind, v. I-II. New York : Harcourt, 1978.

FERRAZ JÚNIOR, Tércio Sampaio. Lafer: a reconstrução dos direitos humanos, in Revista Brasileira de Filosofia, facs. 153, v. XXXVIII, 1989.

LAFER, Celso. A reconstrução dos direitos humanos: um diálogo com o pensamento de Hannah Arendt. São Paulo : Companhia das Letras, 1988.

LAFER, Celso. Hannah Arendt: pensamento, persuasão e poder. Rio de Janeiro : Paz e Terra, 1979. 